dégénérescence axonale [6]. Les colorations immunohistochimiques des souris Irp2 ${ }^{-/-}$, comparées à des témoins, semblent montrer que la ferritine est transportée de la cellule vers les axones (Figure 1). Elle pourrait être dégradée dans les lysosomes à la partie distale des axones, et dans les terminaisons présynaptiques, libérant du fer ferreux, à moins que les hétéropolymères instables libèrent du fer libre dans l'axone ou dans les synapses.

Ajoutons qu'en pathologie humaine, l'acéruloplasminémie, une maladie récessive rare, due à une mutation dans le gène

1. Qian ZM, Shen X. Brain iron transport and neurodegeneration. Trends Mol Med 2001 ; $7: 104-8$.

2. Zhou B, Westaway SK, Levinson B, et al. A novel pantothenate kinase gene (PANK) is defective in Hallervorden-Spatz syndrome. Nat Genet 2001 ; 28 : 345-9. codant pour la céruloplasmine, et caractérisée par une neurodégénérescence associée à un diabète et à une rétinopathie, a été expliquée par une interaction entre la céruloplasmine et la ferritine, et que la protéine responsable de la maladie de Friedreich est, elle aussi, impliquée dans le métabolisme du fer $(\rightarrow)$.

Ainsi, peu à peu, se constitue un faisceau d'arguments en faveur de la relation de cause à effet entre les troubles du transport du fer dans le cerveau et les neurodégénérescences. $\diamond$

Brain iron transport and neurodegeneration

\section{$(\rightarrow) \mathrm{m} / \mathrm{s}$ \\ 1999, n'11, p. 1314}
3. Afshar K, Gonczy P, DiNardo $S$, Wasserman SA. Fumble encodes a pantothenate kinase homolog required for proper mitosis and meiosis in Drosophila melanogaster. Genetics $2001 ; 157: 1267-76$.
4. Heafield MT. Plasma cysteine and sulfate levels in patients
Parkinson's and Alzheimer's disease. Neuroscience 1990 ; $110: 216-20$.
5. Curtis AR, Fey C, Morris CM, et al. Mutation in the gene encoding ferritin light poly- peptide causes dominant adult-onset basal ganglia disease. Nat Genet 2001 ; $28: 350-4$. with motor neurone,

\author{
6. LaVaute $T$, Smith $S$, \\ Cooperman S, et al. Targeted \\ deletion of the gene enco- \\ ding iron regulatory protein- \\ 2 causes misregulation of \\ iron metabolism and neuro- \\ degenerative disease in \\ mice. Nat Genet 2001 ; 27 : \\ 209-14.
}

\title{
NOUVELL
}

\section{Cycline $\varepsilon$ et cancer, une histoire de destruction}

Vjekoslav Dulić qui assurent I'(in)activation ordonnée des Cdk.

Dans ce contexte, le contrôle des quantités de cyclines est d'une extrême importance et les mécanismes impliqués intriguent un bon nombre de

chercheurs depuis une

1919 , route de Mende, 34293 Montpellier, France. décennie. Le taux cellulaire en cyclines est déterminé d'une part au niveau transcriptionnel, et d'autre part par leur destruction, qui est sous la dépendance du système ubiquitine/protéasome, un complexe multiprotéique jouant un rôle majeur dans la protéolyse intracellulaire. La dégradation par ce système se produit en deux étapes : (a) le substrat (souvent phosphorylé) est «marqué » par conjugaison covalente de chaînes d'ubiquitine, ce qui permet sa reconnaissance et (b) la dégradation des molécules polyubiquitinylées par le protéasome 26S [1]. La question suivante se pose alors : comment les protéines 
destinées à la dégradation sont-elles spécifiquement reconnues et adressées vers cette «machinerie de destruction », et ce à des moments précis ? C'est un sujet de recherche intense. Grâce en particulier aux études réalisées dans la levure Saccharomyces cerevisiae, un organisme bien adapté à la manipulation génétique, on connaît bon nombre des composants des complexes (appelés ubiquitine ligases) jouant un rôle dans la reconnaissance du substrat et son adressage vers la destruction. Parmi les ubiquitine ligases [1], SCF joue un rôle essentiel dans le déclenchement de la phase S. SCF est constitué de quatre sous-unités : un cœur composé de Skpl, Cdc53/Cull (Culline) et Rbxl/Rocl, et un membre de la famille des protéines F-box, une sous-unité adaptatrice déterminant la spécificité du substrat (Figure 1). Skp2, une protéine F-box, participe à la dégradation d'un régulateur négatif de la prolifération, p27 $7^{\mathrm{Kipl}}$, un inhibiteur des Cdk dont la destruction en phase Gl favorise l'entrée en phase $S$ [2] (Figure 1). Pour être reconnu par Skp2, p $27^{\text {Kipl }}$ doit d'abord être phosphorylé par le complexe $C d k 2$-cycline $\varepsilon$, élément clé dans le déclenchement de la réplication d'ADN. Parmi les nombreuses cibles de $C d k 2$-cycline $\varepsilon$ figure un suppresseur de tumeur, la protéine du rétinoblasto- me ( $p R b$ ) qui, dans son état actif (non phosphorylé), bloque l'expression des gènes requis pour la synthèse d'ADN (Figure 1).

La dégradation de la cycline $\varepsilon$ en phase $S$ est aussi dépendante d'ubiquitinylation et semble cruciale pour le bon déroulement de la réplication car son expression constitutive provoque une instabilité génomique [3]. Dans plusieurs cancers un taux cellulaire faible de $p 27^{\text {Kipl }}$ a été associé à un mauvais pronostic, et la cycline $\varepsilon$ est surexprimée dans de nombreuses lignées cancéreuses [4] indiquant que la dégradation ordonnée de ces deux régulateurs doit être strictement contrôlée.

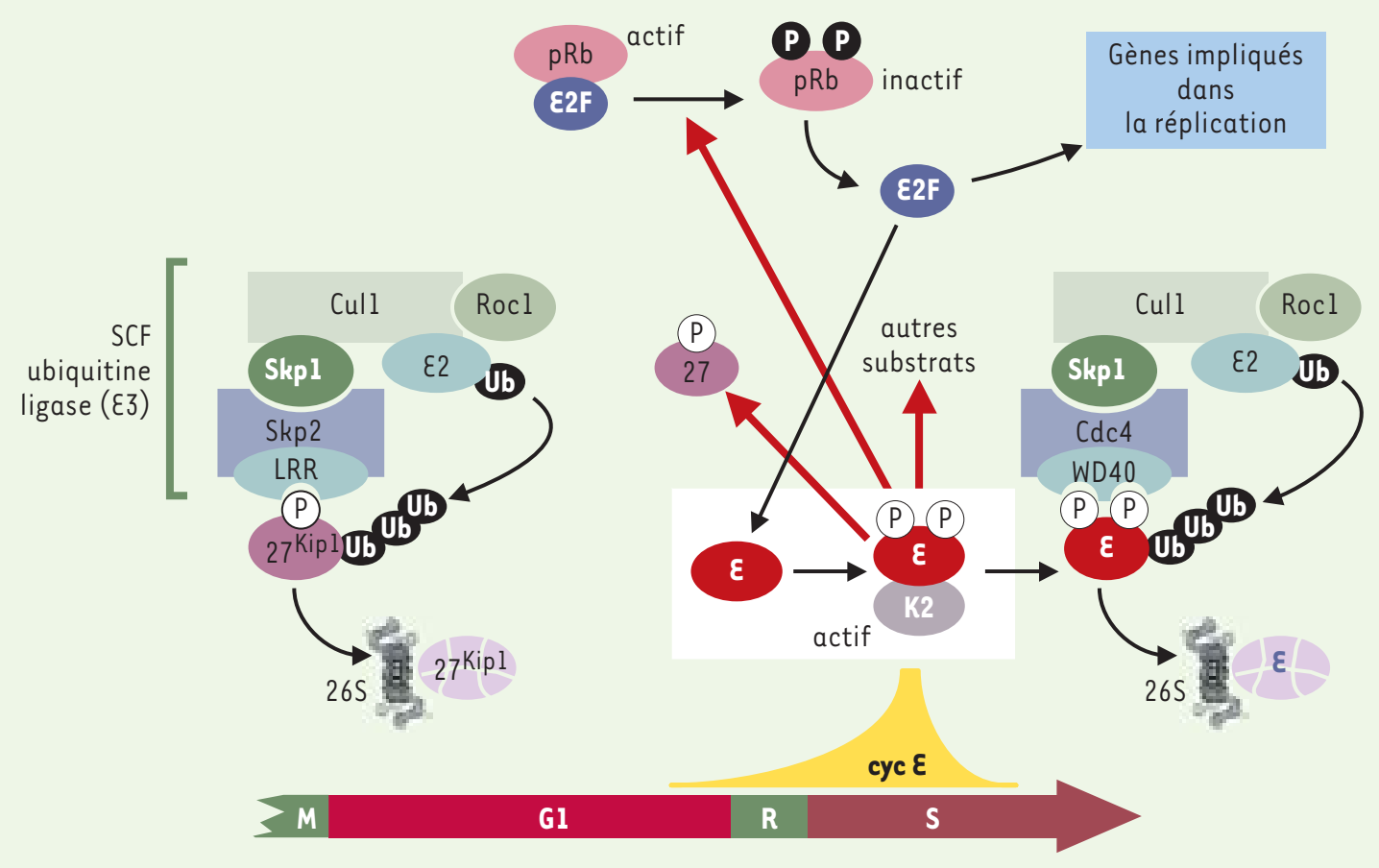

Figure 1. Adressage de la cycline $\varepsilon$ vers la destruction par une ubiquitine ligase.

Le complexe $\mathrm{Cdk2-cycline} \varepsilon$ est un régulateur positif de la duplication du génome. II s'accumule dans le noyau en phase $\mathrm{Gl}$ et son activité est maximale au début de la phase $S$ (transition Gl/S). Ses cibles sont entre autres la protéine du rétinoblastome ( $p R b$ ), un suppresseur de tumeur et régulateur négatif de la prolifération, et un inhibiteur de $C d k, p 27^{\text {Kipl }}$. Après phosphorylation, pRb est inactivée et libère les facteurs de transcription de la famille $\varepsilon 2 F$ qui stimulent l'expression de nombreux gènes (y compris celui de la cycline $\varepsilon$ ) requis pour la réplication. Par ailleurs, p27, une fois phosphorylée, est reconnue par Skp2, une protéine F-box, est ensuite ubiquitinylée par SCF, une ubiquitine ligase (દ3), puis rapidement dégradée par le protéasome $26 \mathrm{~S}$ [1]. En début de phase S, la cycline $\varepsilon$ est phosphorylée par Cdk2 et adressée à l'ubiquitine ligase en se liant à une autre protéine $\mathrm{F}$ box, hCdc4/FbW7/Ago (Cdc4), découverte récemment par trois laboratoires [5-7]. WD40 et LRR sont des domaines des protéines F-box impliqués dans le recrutement spécifique des substrats de SCF. Dans certaines cellules cancéreuses, des mutations dans le domaine WD40 de Cdc4 empêchent la dégradation de la cycline $\varepsilon$, provoquant ainsi sa stabilisation. L'accumulation incontrôlée de la cycline $\varepsilon$ peut perturber l'équilibre existant entre les niveaux de kinase et des inhibiteurs (tels que p $27^{\text {Kipl }}$ ), ce qui engendre un dérèglement de la réplication du génome et, par conséquent, de la prolifération cellulaire. 
La protéine F-box dirigeant la cycline $\varepsilon$ vers la voie de destruction vient d'être identifiée par trois laboratoires américains. Bien que certaines indications antérieures suggéraient que Skp2 pouvait être impliquée dans cet adressage, ces chercheurs ont réussi à démontrer que c'est, en fait, la protéine $\mathrm{Cdc} 4 / \mathrm{Fbw} 7 /$ Ago qui joue ce rôle, et que la mutation de cette protéine pourrait être à l'origine d'un développement tumoral [5-7]. Pour y parvenir, deux laboratoires, celui de S.I. Reed (Scripps Institute, La Jolla, CA, USA) et celui de S.J. Elledge (Baylor College, Houston, TX, USA), ont utilisé comme modèle la levure S. cerevisiae, dont les voies de protéolyse sont bien caractérisées. De plus, comme la cycline $\varepsilon$ humaine peut se substituer dans la levure aux cyclines Gl endogènes [8], on pouvait s'attendre à ce que la levure puisse régler l'expression et l'activité de cette cycline. En étudiant des cellules de levure dont les gènes codant pour les divers composants du complexe SCF ont été mutés, ces chercheurs ont découvert que la cycline $\varepsilon$ était stabilisée dans les cellules ayant une mutation dans le gène $c d c 4[5,6]$. Ensuite, ils ont pu montrer in vitro que son orthologue humain, hCdc4 [5] ou Fbw7 [6], en s'associant à la cycline $\varepsilon$ préalablement phosphorylée (probablement par (dk2), catalyse son ubiquitinylation et, par conséquent, sa destruction. Des mutations dans le domaine WD40 de hCdc4/Fbw7 (Figure I) empêchent son association avec la cycline $\varepsilon$ phosphorylée, indiquant que ce domaine est nécessaire à la reconnaissance du substrat. Ces résultats ont aussi été validés dans des cellules humaines dans lesquelles la surexpression de hCdc4 conduit à une diminution de la demi-vie de la cycline $\varepsilon$ tandis que la présence d'une forme mutée de cette protéine provoque sa stabilisation.

L'équipe de I.K. Hariharan (MGH Cancer Center, Charlestown, USA), qui, elle, recherchait un environnement génétique favorisant la prolifération de cellules de drosophile, observait au même moment que la cycline $\varepsilon$ était très stabilisée si on mutait le gène archipelago (ago, un orthologue de $(d c 4)$ dans les cellules [7]. Contrairement aux cellules avoisinantes de type sauvage qui se trouvaient en état de quiescence, les cellules mutantes continuaient à proliférer, démontrant ainsi qu'une dégradation inefficace de la cycline $\varepsilon$ peut stimuler la division cellulaire. Encore plus intéressants sont les résultats montrant que plusieurs lignées d'origine tumorale exprimant des taux élevés de cycline $\varepsilon$ présentent justement des mutations dans le domaine WD40 de hCdc4/ Fbw7/Ago. Sachant que l'instabilité du génome provoquée par un dérèglement de la cycline $\varepsilon$ peut engendrer des changements favorisant la cancérogenèse, il est possible que hCdc4/Fbw7/Ago soit un suppresseur de tumeur, en d'autres termes un régulateur négatif de la prolifération. Enfin, il a été montré que l'orthologue de Cdc4 chez le nématode Caenorhabditis elegans, SEL-10, est impliqué dans la dégradation des présénilines (des protéines codées par les gènes dont les mutations sont à l'origine de la maladie d'Alzheimer) [9], ainsi que des oncogènes de la famille Notch [10], jouant un rôle important dans la signalisation cellulaire. Et ce n'est sûrement pas la fin de l'histoire. $\diamond$

Cyclin $\varepsilon$ and cancer: a trash story
1. Coux 0 , Piechazyk M. Le système ubiquitine/ protéasome : un ensemble (de) complexe(s) pour dégrader les protéines. Med Sci 2000 ; $5: 623-9$.

2. Sutterluty $H$, Chatelain $\varepsilon$, Marti A, et al. p45S KP2 promotes $p 27^{\text {Kipl }}$ degradation and induces $S$ phase in quiescent cells. Nat Cell Biol 1999 ; 1 : 207-14.

3. Spruck CH, Won KA, Reed SI. Deregulated cyclin $\varepsilon$ induces chromosome instability. Nature 1999 ; 401 : 297-300.

4. Keyomarsi K, Herliczek TW. The role of cyclin $\varepsilon$ in cell proliferation, development and cancer. Prog Cell Cycle Res 1997 ; 3 : 171-91.

5. Strohmaier H, Spruck CH, Kaiser P, Won KA, Sangfelt O, Reed SI. Human F-box protein hCdc4 targets cyclin $\varepsilon$ for proteolysis and is mutated in a breast cancer cell line. Nature 2001 ; 413 : 316-22.

6. Koepp DM, Schaefer LK, Ye X, et al. Phosphorylationdependent ubiquitination of cyclin $\varepsilon$ by the SCF Fbw7 ubiquitin ligase. Science 2001 ; 294 : 173-7.

7. Moberg KH, Bell DW, Wahrer DC, Haber DA, Hariharan IK. Archipelago regulates cyclin $\varepsilon$ levels in Drosophila and is mutated in human cancer cell lines. Nature 2001 ; 413 : 311-6.

8. Lew DJ, Dulic V, Reed SI. Isolation of three novel human cyclins by rescue of $\mathrm{Gl}$ cyclin (Cln) function in yeast. Cell 1991 ; 66 : $1197-$ 206.

9. Wu G, Hubbard EJ, Kitajewski JK, Greenwald I. Evidence for functional and physical association between Caenorhabditis elegans SEL-10, a Cdc4p-related protein, and SEL-12 presenilin. Proc Natl Acad Sci USA 1998 ; 95 : 15787-91.

10. Gupta-Rossi N, Le Bail 0, Gonen $\mathrm{H}$, et al. Functional interaction between SEL-10, an F-box protein, and the nuclear form of activated Notchl receptor. J Biol Chem 2001 ; 276 : 34371-8. 